\title{
A REVERSIBLE DEVELOPMENTAL CHANGE IN THE ABILITY OF CILIARY GANGLION NEURONS TO EXTEND NEURITES IN CULTURE $^{1}$
}

\author{
FRANK COLLINS ${ }^{2}$ AND MARK R. LEE \\ Department of Anatomy, University of Utah School of Medicine, Salt Lake City, Utah 81112
}

Received August 28, 1981; Revised November 10, 1981; Accepted November 23, 1981

\begin{abstract}
From stages 35 to 40 in the chicken embryo, ciliary ganglion neurons undergo a developmental change which is detected in our assay system as a marked decline in the ability to extend neurites when placed in culture. This developmental loss is observed when the neurons are placed in culture as single, dissociated cells or as undissociated ganglion explants. The loss of the ability to extend neurites in culture is not a transitory phenomenon, for no recovery of this ability is observed during the rest of embryonic development or posthatching. There is a close temporal correlation between the embryonic period during which the ability of ciliary ganglion neurons to extend neurites declines and the period (stages 35 to 40) when these neurons form functional peripheral synapses in the embryo. The ability to extend neurites rapidly is not recovered during normal development as long as the ganglion and its connections within the organism remain intact. However, if the ciliary ganglia are removed from a stage 40 embryo and cultured for 3 to 4 days before being dissociated, the neurons have largely recovered their ability to initiate neurite outgrowth rapidly. This recovery after removal from the embryo is also presumably responsible for the eventual dense outgrowth of neurites from stage 40 ganglion explants after a 3- to 4-day delay in culture. Our results, in conjunction with similar results from other systems, suggest a cause and effect relationship between the establishment or disruption of peripheral connections and the loss or recovery, respectively, of the ability to extend neurites rapidly.
\end{abstract}

In order to use tissue culture to study neural development, it is desirable to have a homogeneous source of neurons which go through a synchronous and well studied developmental sequence in the embryo. Thus, one is able to obtain a population of neurons for culturing which are at the same known developmental stage in order to study their behavior and subsequent development in vitro. The avian ciliary ganglion comes close to being such a source of staged neurons. The ganglion is composed of a relatively homogeneous population of motoneurons. These are subdivided into two groups, choroid and ciliary, which differ in some of their physiological properties and in the location within the eye of their target muscles (Marwitt et al., 1971; Pilar et al., 1980). Developmental events, such as the formation of preganglionic and postganglionic synapses and the developmental death of neurons within the ganglion, occur

\footnotetext{
${ }^{1}$ We wish to thank.James F. Garrett, Jr. for his assistance in several of these experiments. This work was supported by Grant NS15130 from the National Institutes of Health.

${ }^{2}$ To whom correspondence should be addressed.
}

within defined embryonic periods which are approximately the same for all neurons in the ganglion (Landmesser and Pilar, 1974; Pilar et al., 1980).

An important advance in exploiting this system came when conditions were first described for culturing dissociated neurons of the chick embryo ciliary ganglion (Helfand et al., 1976). Much effort has subsequently gone into refining culture conditions and studying the effects on neural development in vitro of various of the extrinsic culture conditions (Nishi and Berg, 1977; Tuttle et al., 1980; Varon et al., 1979). The system also has been used to demonstrate that the tendency of ciliary ganglion neurons to die at a particular time in development is not an intrinsic property of the neurons, since dissociated neurons placed in culture before the time of cell death remain alive in vitro (Nishi and Berg, 1977, 1979). In contrast, the development of several properties of ciliary ganglion neurons, such as increased cell body diameter and increased levels of transmitter-related enzymes, appear on schedule in dissociated cell cultures (Nishi and Berg, 1979).

In our own study of the factors controlling neurite 
outgrowth from dissociated ciliary ganglion neurons (Collins, 1978a, b, 1980; Collins and Garrett, 1980), we observed a marked difference in the ability of neurons from different stage embryos to initiate neurite outgrowth in culture. As the following observations indicate, it has proved possible to make a reasonable correlation between this developmental change in ability to extend neurites and the formation of peripheral connections by the neurons in vivo.

\section{Materials and Methods}

White Leghorn chicken eggs incubated at $39^{\circ} \mathrm{C}$ in a humidified atmosphere were used. The embryos were staged by reference to the criteria of Hamburger and Hamilton (1951). The results are reported in terms of embryonic stage; for conversion to days of incubation: stage 35 corresponds to approximately 9 days of incubation, while stage 40 corresponds to approximately 14 days. Heart cell-conditioned medium was produced by monolayer cultures of 10-day chick embryo heart cells as previously described (Collins, 1978a). Heart cell microexudate was prepared as previously described (Collins, 1980 ) by treating a confluent monolayer of 10-day chick embryonic heart cells grown on plastic culture dishes with EGTA to remove the cells, followed by several washes of the dishes with distilled water.

Ciliary ganglia were removed from the embryos and either dissociated into single cells or cultured as undissociated explants. For explant cultures, whole ganglia were cut into two to four smaller pieces with iridectomy knives. Dissociation into single cells was accomplished using trypsin in $\mathrm{Ca}^{2+}, \mathrm{Mg}^{2+}$-free Hanks' balanced salt solution as previously described (Collins, 1978a). Dissociated cells were incubated in culture medium (see below) in plastic tissue culture dishes for $3.5 \mathrm{hr}$ before being plated out under growth conditions. This was done in order to separate the non-neuronal cells in the ganglion, which adhered to the tissue culture dishes, from the neurons, which did not adhere and could be transferred to the growth dishes.

Culture conditions. A culture substratum suitable for neurite outgrowth was prepared as follows: plastic tissue culture dishes (Falcon) were coated with polyornithine as previously described (Collins, 1978a). The polyornithine-coated dishes then were incubated overnight with heart cell-conditioned medium in order to pre-coat the dish with a substratum-conditioning factor which induces neurite outgrowth from ciliary ganglion neurons (Collins, $1978 \mathrm{~b})$. The dishes then were washed and dissociated neurons or undissociated ganglion pieces were plated on in culture medium. The culture medium was adapted from the one developed by Tuttle et al. (1980) to maintain dissociated ciliary ganglion neurons. This medium consisted of Ham's F12 medium (substituted for minimal essential medium), with $10 \%$ heat-inactivated horse serum and $10 \%$ chick embryo extract. Cultures were incubated at $37^{\circ} \mathrm{C}$ in a humidified atmosphere with sufficient $\mathrm{CO}_{2}$ added to maintain the $\mathrm{pH}$ between 7.2 and 7.4.

Assay of neurite outgrowth. In dissociated cell cultures, neurite outgrowth is reported as the increase in the percentage of initiated neurons with time after plating. An initiated neuron is defined as one bearing at least one neurite greater than $15 \mu \mathrm{m}$ in length. This measurement tends to minimize the differences between stage 35 and stage 40 neurons, since it fails to take into account the much greater length of neurites extended by the younger neurons.

When undissociated ganglion pieces were cultured, migration of non-neuronal cells from the explant out onto the polyornithine substratum was very limited so that each ganglion was surrounded by neurites growing directly on the culture substratum with only infrequent non-neuronal cells scattered among the neurites. In some experiments, the metabolic inhibitors 5-fluoro- 2 -deoxyuridine $(10 \mu \mathrm{M})$ and $1-\beta$-D-arabinofuranosylcytosine (10 $\mu \mathrm{M})$ were added to reduce even further the numbers of non-neuronal cells found outside of the ganglion (Estridge and Bunge, 1978). The differences in neurite outgrowth between ganglia of different stages were the same in the presence or absence of these inhibitors.

To obtain an estimate of the density of neurite outgrowth from ganglion pieces of different ages, a scale of 0 to 4 was established in which the densest outgrowth was assigned a value of 4 , and barely detectable outgrowth was assigned a value of 0.5 . For assignment of these numerical values, the outgrowth from an explant was photographed and compared to standard photographs. The density of outgrowth around each explant was typically quite uniform, but in those cases where it was not, the area of densest outgrowth was used for assignment of a numerical value. To give some idea of the density of outgrowth corresponding to these values, a value of 1 is illustrated in Figure $4, B$ and $D ; 2$ in Figure $4 A$; and 4 in Figure $4, C, E$, and $F$.

\section{Results}

Between stages 35 and 40 of embryonic development in the chicken, there is a marked loss in the ability of ciliary ganglion neurons to initiate neurite outgrowth rapidly when dissociated into single cells and placed in culture. Figure 1 compares the time course of neurite initiation in two cultures of dissociated ciliary ganglion neurons, one taken from stage 33 embryos before the developmental change occurs and the other taken from stage 40 embryos just after the change is complete. In both cases, a maximum percentage of initiated neurons is reached within 2 to $3 \mathrm{hr}$ after plating, and this percentage does not increase significantly thereafter. With stage 40 neurons, a maximum of only $19 \pm 7 \%$ (six experiments) are able to extend neurites compared with $78 \pm 18 \%$ for the stage 33 neurons. Not only do a much greater percentage of stage 33 neurons iniliate outgrowth but their neurites are substantially longer and continue to elongate actively, whereas the stage 40 neurites are much shorter and elongate slowly if at all.

At each development stage from stages 35 to 40 , the neurons fall into two classes: (1) those which can readily initiate neurite outgrowth, resembling in this respect stage 35 or younger neurons, and (2) those which extend no or only very short neurites. By stage 40 , the percentage of neurons in class 1 has decreased to almost 0 (Fig. 2). Dissociated ciliary ganglion neurons from stage $44 \mathrm{em}-$ bryos (approximately 18 days of incubation') and from chickens at 2 weeks posthatching are as inactive with 


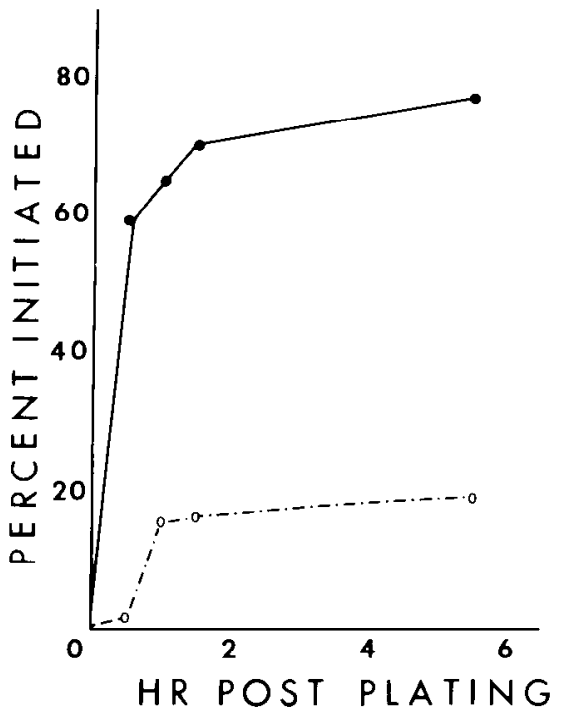

Figure 1. Difference in the time course of neurite initiation between stage 35 and stage 40 neurons. The increase in the percentage of initiated neurons with time after plating is shown for dissociated ciliary ganglion neurons obtained from stage 35 (O) and stage $40(O)$ embryos.

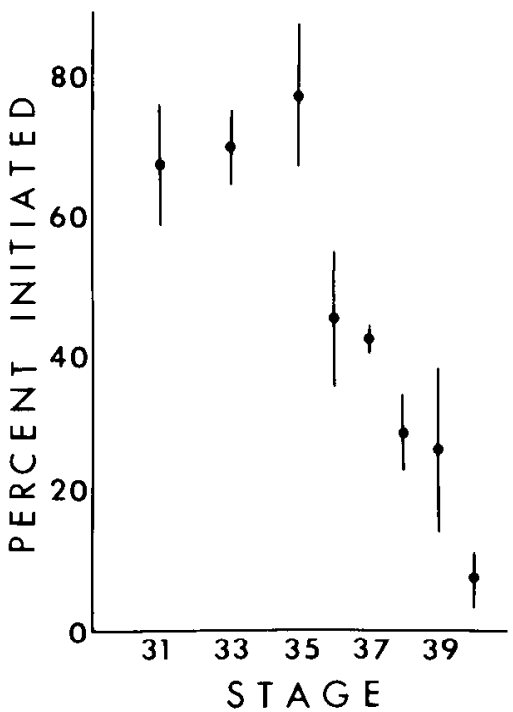

Figure 2. Stage-dependent changes in the extent of neurite outgrowth. The maximum percentage of initiated neurons achieved by each culture of dissociated ciliary ganglion neurons is plotted against the stage embryo from which the neurons were obtained. The results plotted are the mean values and range for three separate experiments.

respect to neurite outgrowth as are stage 40 neurons. Thus, there is no apparent recovery of function during normal development after stage 40.

We have not observed by phase contrast microscopy any obvious physical damage during dissociation to the neurons from stage 40 or older embryos which could explain their inability to extend neurites. Also, in the experiment described in Figure 2, $68 \pm 19 \%$ of the neurons known to be present in the ganglion at each embryonic stage (Landmesser and Pilar, 1974) were recovered after dissociation, preplating, and final culturing. This suggests that there was no undue loss of neurons during the dissociation of older ganglia. Other observations, which are presented in detail below, support the conclusion that the observed developmental change is not an artifact of dissociation: (1) whole, undissociated ganglia show a loss in ability to extend neurites in culture readily between stages 35 and 40 , which parallels that observed with dissociated neurons, and (2) after suitable experimental manipulation, stage 40 ganglia, when dissociated as above, contain a greatly increased proportion of neurons able to extend neurites rapidly.

To determine to what extent our particular culture conditions are responsible for the preceding result with dissociated neurons, neurite outgrowth from stage 35 and 40 neurons was compared using a substratum and culture medium different from that used in our standard protocol. The substratum used was heart cell microexudate (Collins, 1980; Hawrot, 1980), which has been shown to support neurite outgrowth from a variety of dissociated embryonic neurons. The culture medium was whole heart cell-conditioned medium, which has been used to maintain dissociated ciliary ganglion neurons in culture (Helfand et al., 1976; Collins, 1980). Under these conditions, stage 35 neurons attained a maximum of $84 \pm 7 \%$ initiated neurons (in three experiments) compared to $13 \pm 9 \%$ for stage 40 neurons. Therefore, the difference in ability to extend neurites in culture between stage 35 and 40 neurons is manifested in two different culture systems.

Whole ganglia. Undissociated ciliary ganglion explants from embryos of different ages were cultured in order to eliminate the possible effects of dissociation into single cells on the results described above. In these experiments, each ganglion was cut into two to four smaller pieces in order to promote rapid adhesion of the pieces to the substratum and, by exposing the interior of the ganglion, to prevent any connective tissue which

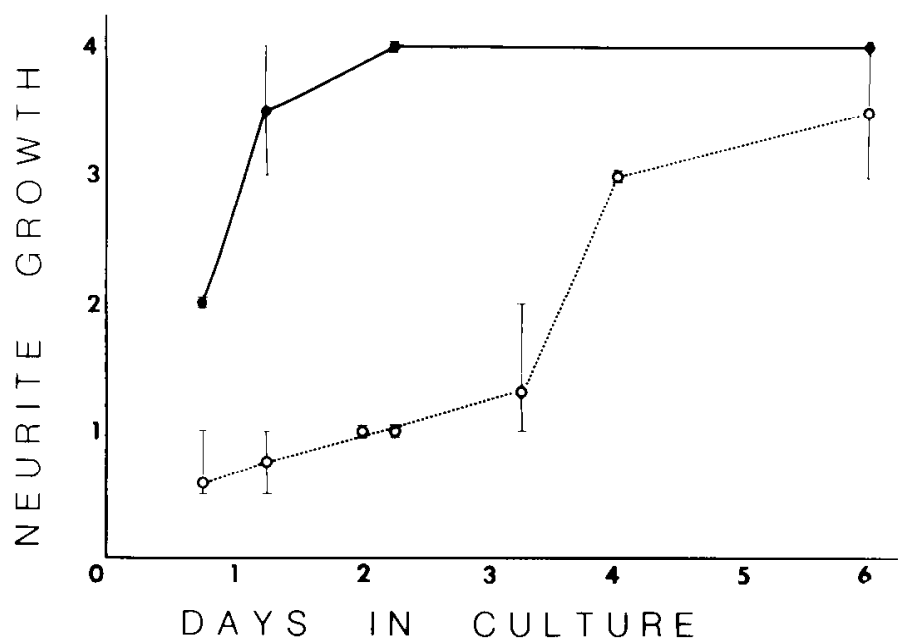

Figure 3. The time course of neurite outgrowth from stage 35 and 40 ciliary ganglion explants. The relative density of neurite outgrowth was estimated at various times after plating for undissociated ciliary ganglion pieces from stage $35(O)$ and stage $40(\mathrm{O})$ embryos. The results plotted are the mean and range for four ganglion pieces from each embryonic stage. 

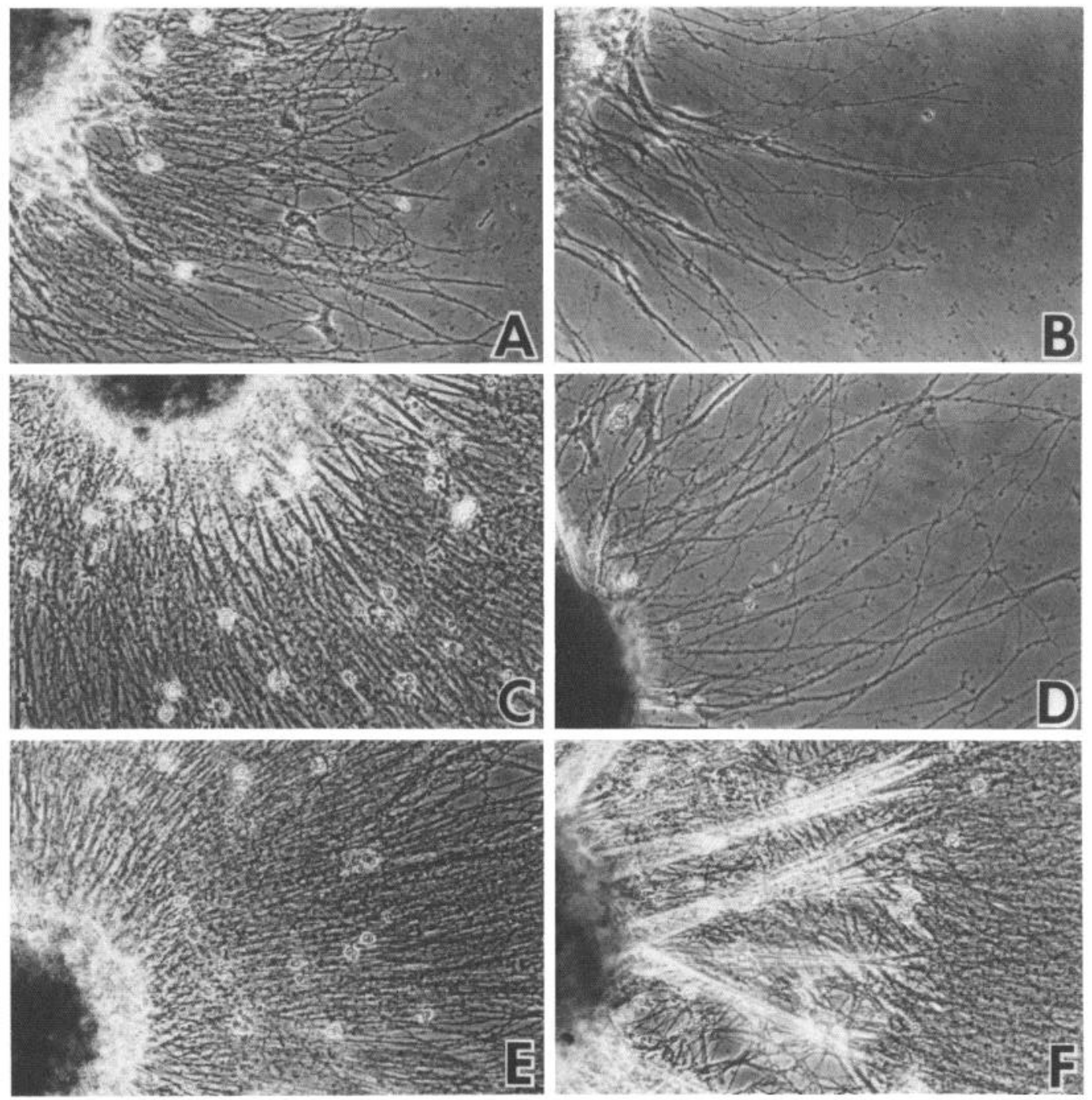

Figure 4. A comparison of neurite outgrowth from stage 35 and 40 ganglion explants. Phase contrast micrographs of ciliary ganglia from stage $35(A, C$, and $E)$ and stage $40(B, D$, and $F)$ embryos. The micrographs were taken after $18(A$ and $B), 54(C$ and $D)$, and $144 \mathrm{hr}(E$ and $F)$ in culture. Magnification $\times 133$.

might be on the exterior of the ganglion from interfering with neurite outgrowth.

Figure 3 compares the time course of neurite outgrowth from stage 35 and 40 ciliary ganglion explants, using a semiquantitative assay of the density of outgrowth which is described under "Materials and Methods." Figure 4 is a set of phase contrast micrographs of a stage 35 and stage 40 explant after various times in culture, illustrating the differences in outgrowth between these two embryonic stages.

The essential differences observed in neurite outgrowth between stage 35 and 40 ganglion explants are the following: after 24 to $36 \mathrm{hr}$ in culture, the stage 35 explant is surrounded by an essentially confluent carpet of neurites (Figs. 3 and $4 C$ ). In contrast, after $24 \mathrm{hr}$ in culture, there is some outgrowth from the stage 40 ganglion, but there is no marked increase in the amount of outgrowth until after $72 \mathrm{hr}$ (Figs. 3 and $4, B$ and $D$ ). Only after 96 to $144 \mathrm{hr}$ in culture does the stage 40 explant produce a confluent carpet of neurites (Figs. 3 and $4 F$ ).
This eventual outgrowth from stage 40 ganglia, which begins after 72 to $96 \mathrm{hr}$ in culture, apparently represents a recovery of the ability of stage 40 neurons to extend neurites as discussed below.

Recovery after removal from the embryo. The observation that stage 40 ciliary ganglion explants eventually extended a dense network of neurites after 3 to 4 days of relative inactivity in culture suggests the possibility that, after removal from the embryo, the stage 40 neurons undergo a gradual recovery of the ability to extend neurites. To test this, stage 40 ciliary ganglia were cultured as above for $72 \mathrm{hr}$ and then dissociated by the usual method into single cells. At the same time, freshly dissected stage 40 and 44 (96-hr-older) ganglia were dissociated as controls. As can be seen in Figure 5, neurons from ganglia which had been removed from the embryo 3 days previously initiate neurite outgrowth to a much greater extent than do neurons from control ganglia. Not only are a high proportion of the neurons from previously explanted ganglia able to initiate outgrowth rapidly but 


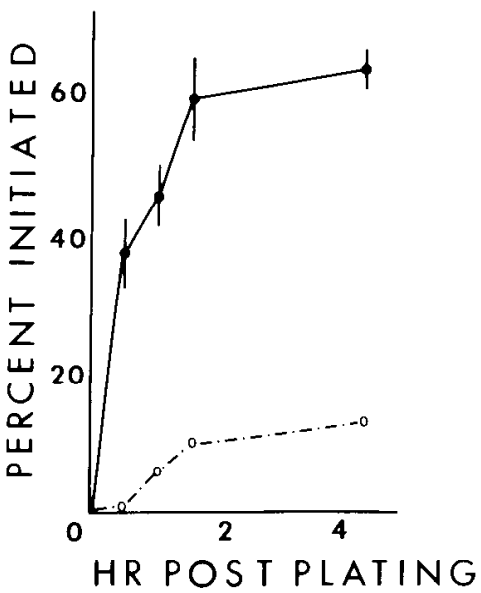

Figure 5. Recovery by stage 40 neurons of the capacity to extend neurites. The increase in the percentage of initiated neurons with time after plating is shown for dissociated stage 40 ciliary ganglion neurons prepared as follows: 0 , three stage 40 ganglia were cultured for $72 \mathrm{hr}$, and then the ganglia were scraped from the dish with a sterile rubber policeman, dissociated into single cells, and placed in culture. $O$, Three ganglia were dissected from stage 40 embryos, immediately dissociated into single cells, and cultured. The results plotted are the mean values and range for two separate experiments.

neurite lengths and numbers per cell are comparable to those observed for younger stage 33 to 35 neurons.

This recovery of function in precultured stage 40 ganglia is not dependent on the neurons having extended neurites in culture before dissociation. By $72 \mathrm{hr}$, there has been relatively little outgrowth from the ganglion explant. Also, in one experiment in which stage 40 ciliary ganglia were kept in suspension culture and therefore were unable to extend neurites for $72 \mathrm{hr}$ before being dissociated, the neurons demonstrated rapid neurite outgrowth compared to freshly dissected and dissociated controls.

The number of neurons recovered after dissociation of precultured stage 40 ganglia (2048 \pm 312 neurons/ganglion) is approximately the same as the number recovered from control stage 40 ganglia $(2310 \pm 220$ in two experiments). Therefore, it can be concluded that the higher proportion of neurons capable of initiating outgrowth in the previously explanted ganglia is not due to selection or differential survival of a more active subpopulation of neurons.

It is worth noting that we have not observed an analogous delayed extension of neurites from single, dissociated stage 40 neurons in culture. This is possibly a result of the fact that only approximately $20 \%$ of the dissociated neurons appear intact in our culture system at $72 \mathrm{hr}$ and only 5 to $10 \%$ at $96 \mathrm{hr}$. Since the older dissociated neurons die so rapidly in our culture conditions, it is not possible to say at present if isolated stage 40 neurons are capable of eventually extending neurites as happens with undissociated ganglia. As a consequence of this, it is not possible to rule out a contribution of the non-neuronal cells within the ganglion explants to the recovery of function in the stage 40 neurons.

\section{Discussion}

The present results demonstrate that, from stages 35 to 40 in the chicken embryo, there is a steady decrease in the ability of ciliary ganglion neurons to initiate neurite outgrowth rapidly when placed in culture. This conclusion is supported by our results using both single, dissociated neurons and undissociated ganglion explants. This same result also was observed using two different culture substrata and two different culture media. Previously, both Ebendal (1979) and McLennan and Hendry (1980) have reported a sharp decrease, which is near maximal by stage 40 , in the ability of chick embryo ciliary ganglion explants of increasing embryonic ages to extend neurites in culture. Ebendal (1979) used ganglia co-cultured with heart explants in a collagen gel, while McLennan and Hendry (1980) used ganglia cultured on collagen in medium containing rat heart extract. Our similar results from ciliary ganglion explants were obtained by using a polyornithine substratum precoated with conditioned medium in medium containing chick embryo extract. Thus, the same apparent loss by embryonic stage 40 in the ability of ciliary ganglion neurons to extend neurites in culture rapidly has been observed in a wide variety of culture conditions, all of which are able to support rapid and extensive neurite outgrowth from the younger embryonic stages.

At present, it seems to us that there are two possible explanations for this result. One is that there is a change during development in the requirements of ciliary ganglion neurons for trophic support. Therefore, given the appropriate, as yet undiscovered, factor in their medium, even stage 40 ciliary ganglion neurons would be capable of rapid and extensive neurite outgrowth. The second explanation is that there is a loss by stage 40 in the inherent capacity of ciliary ganglion neurons to extend neurites due, as one possible example, to altered metabolic patterns which result in decreased production of the cytoskeletal elements necessary for neurite extension. The only evidence which we have at present bearing on a choice between these alternatives is that, in our culture conditions, using dissociated neurons, neurite outgrowth begins within $30 \mathrm{~min}$ of plating. This can even occur in Hanks' balanced salt solution in the absence of added trophic factors from serum, tissue extracts, or conditioned medium (F. Collins, unpublished observation). These observations suggest that, in these assay conditions, outgrowth is so rapid that trophic or survivalpromoting components are unnecessary and that one is actually measuring the inherent capacity of the neurons to extend neurites. If so, this would support the second explanation that the developmental change that we have observed is due to a loss of the inherent capacity of the neurons to initiate neurite outgrowth readily.

It has been reported that chick dorsal root ganglion explants undergo a developmental loss in ability to extend neurites in culture (Herrup and Shooter, 1975; Winick and Greenberg, 1965; Greene, 1977). This loss may be similar in nature to the developmental change that we have described for neurons of the ciliary ganglion. However, the developmental change in dorsal root ganglion neurons occurs approximately 2 days later in embryonic development than the analogous change in ciliary ganglion neurons. These results suggest that different classes of embryonic neuron may go through a similar loss in ability to initiate neurite outgrowth readily, with each type of neuron going through this change during a char- 
actcristic and different period of embryonic development. Reversibility of the developmental stage. The loss by stage 40 in the capacity to initiate outgrowth in culture is not a transitory loss but an apparently permanent change since we have observed no recovery of function during later embryonic development or posthatching. That is, the loss is permanent as long as the ganglion and its connections within the embryo remain intact. After removal from the embryo, we have found that stage 40 neurons undergo a gradual recovery in their ability to initiate outgrowth in culture rapidly. Thus, under these circumstances, stage 40 neurons can extend neurites with approximately the same vigor as younger embryonic neurons.

Relationship to synapse formation. The loss during development in the ability of ciliary ganglion neurons to initiate outgrowth seems to be related to the formation of peripheral synaptic connections. The period from stages 35 to 40 , during which ciliary ganglion neurons undergo the loss in ability to extend neurites in culture readily, is the same period during which the ciliary ganglion is undergoing other important developmental changes. Our observations add to the list of events now known to occur in the ciliary ganglion during this critical period of its development. Stages 35 to 40 encompass the period during which approximately $50 \%$ of all of the neurons in the ganglion die (Landmesser and Pilar, 1974). This is also the period during which the ciliary neuron subpopulation of the ciliary ganglion form functional synapses on their periphery (Pilar et al., 1980). Although the time of synapse formation by the choroid neurons in the ganglion has not been studied, from the close relationship normally observed between the period of neuronal cell death and the period of synapse formation, it is reasonable to assume that, between stages 35 and 40 , functional synapses are formed by the entire ganglion population (ciliary and choroid neurons). Therefore, from stages 35 to 40 , among surviving neurons, there is a steady increase in the proportion which have made functional peripheral connections. The neurons undergoing cell death in the ganglion are apparently lost during dissociation in our experiments, since the number of intact neurons recovered from each ganglion decreases from stages 35 to 40 by approximately the expected $50 \%$. Those neurons which are recovered appear to us to fall into two classes: those which can extend neurites as readily as stage 35 or younger neurons and those which can extend no or only very short neurites. The proportion of the former decreases to almost none by stage 40 . There is thus a close correlation between the decrease in the proportion of neurons able to initiate outgrowth and the increase in the proportion of neurons which have formed functional synapses during this period (compare our Fig. 2 and Fig. 7 in Landmesser and Pilar, 1974).

A relationship between the possession of intact peripheral connections and the reduced ability to extend neurites in culture has been reported previously for retinal ganglion neurons in retinal explants of goldfish and frogs (Agranoff et al., 1976; Johns et al., 1978; Landreth and Agranoff, 1979). In these experiments, rapid neurite outgrowth was observed in culture from the retinal ganglion cells in retinal explants taken from Xenopus embryos too young to have formed tectal connections, whereas retinal explants taken from later larval stages showed delayed neurite outgrowth (Agranoff et al., 1976). Prior axotomy, by optic nerve lesion, greatly increased the rapidity and extent of neurite outgrowth from the retinal ganglion cells in the late larval explants, making them more closely resemble the embryonic neurons (Landreth and Agranoff, 1979).

Our observations on the ciliary ganglion are essentially similar except that we have used single neurons as well as whole explants and have observed a close temporal correlation between peripheral synapse formation and the loss of ability to initiate neurite outgrowth. The recovery of function that we have observed in stage 40 neurons several days after removal from the embryo is presumably a result of having broken their peripheral connections. Such prior axotomy, as with the larval retinal explants, restores to the stage 40 ciliary ganglion neurons a capacity to initiate neurite outgrowth in culture which is similar to that observed in stage 35 or younger neurons.

The reversible developmental change which ciliary ganglion and retinal ganglion neurons undergo in their ability to initiate neurite outgrowth may be related to the capacity to regenerate axons in vivo after nerve injury. This seems to be the case for retinal ganglion neurons. If retinal ganglion neurons are disconnected from their periphery by a lesion to the optic nerve applied several days before the same animals are given a second, more proximal, test lesion to the nerve, regenerating axons appear in response to the second lesion twice as rapidly as in control animals not given the previous "conditioning" lesion (McQuarrie and Grafstein, 1981). Presumably, the conditioning lesion triggers the reversion of retinal ganglion neurons to a state resembling that which they possessed embryonically before forming functional synapses, thereby accelerating the regenerative response to the subsequent test lesion. This reversion of the neurons also may be a normal part of the regenerative process made visible in the well known cytological changes undergone by the nerve cell body after axotomy (Grafstein and McQuarrie, 1978). These changes presumably reflect conversion of the neurons to a state more suitable for supporting neurite outgrowth.

The nature of the changes in nerve cells during development which limits their ability to extend axons as well as the mechanism by which synapse formation apparently signals these changes are subjects requiring further investigation. Such studies might provide useful means for promoting the regeneration of damaged nerves by allowing one to stimulate at will the reversion of neurons to a more "embryonic" condition.

\section{References}

Agranoff, B. W., P. Field, and R. M. Gaze (1976) Neurite outgrowth from explanted Xenopus retina: An effect of prior optic nerve section. Brain Res. 113: 225-234.

Collins, F. (1978a) Axon initiation by ciliary neurons in culture. Dev. Biol. 65: 50-57.

Collins, F. (1978b) Induction of neurite outgrowth by a conditioned-medium factor bound to the culture substratum. Proc. Natl. Acad. Sci. U. S. A. 75: 5210-5213.

Collins, F. (1980) Neurite outgrowth induced by the substrate associated material from nonneuronal cells. Dev. Biol. 79: 247-252. 
Collins, F., and J. E. Garrett, Jr. (1980) Elongating nerve fibers are guided by a pathway of material released from embryonic nonneuronal cells. Proc. Natl. Acad. Sci. U. S. A. 77: 62266228.

Ebendal, T. (1979) State-dependent stimulation of neurite outgrowth exerted by NGF and chick heart in cultured embryonic ganglia. Dev. Biol. 72: 276-290.

Estridge, M., and R. Bunge (1978) Compositional analysis of growing axons from rat sympathetic neurons. J. Cell Biol. 79: $138-155$.

Grafstein, B., and I. G. McQuarrie (1978) Role of the nerve cell body in axonal regeneration. In Neuronal Plasticity, C. W. Cotman, ed., pp. 155-195, Raven Press, New York.

Greene, L. A. (1977) Quantitative in vitro studies on the nerve growth factor (NGF) requirement of neurons. Dev. Biol. 58: 106-113.

Hamburger, V., and H. L. Hamilton (1951) A series of normal stages in the development of the chick embryo. J. Morphol. 88: 49-92.

Hawrot, E. (1980) Cultured sympathetic neurons: Effects of cell-derived and synthetic substrata on survival and development. Dev. Biol. 74: 136-151.

Helfand, S. L., G. A. Smith, and W. K. Wessells (1976) Survival and development in culture of dissociated parasympathetic neurons from ciliary ganglia. Dev. Biol. 50: 541-547.

Herrup, K., and E. M. Shooter (1975) Properties of the nerve growth factor receptor in development. J. Cell Biol. 67: 118125.

Johns, P. R., A. M. Heacock, and B. W. Agranoff (1978) Neurites in explant cultures of adult goldfish retina derived from ganglion cells. Brain Res. 142: 531-537.
Landmesser, L., and G. Pilar (1974) Synaptic transmission and cell death during normal ganglionic development. J. Physiol. (Lond.) 241: 737-749.

Landreth, G. E., and B. W. Agranoff (1979) Explant culture of adult goldfish retina: $A$ model for the study of CNS regeneration. Brain Res. 161: 39-53.

Marwitt, R., G. Pilar, and J. N. Weakly (1971) Characterization of two ganglion cell populations in avian ciliary ganglia. Brain Res. 25: 317-334.

McLennan, I. S., and I. A. Hendry (1980) Influence of cardiac extracts on cultured ciliary ganglia. Dev. Neurosci. 3: 1-10.

McQuarrie, I. G., and B. Grafstein (1981) Effect of a conditioning lesion on optic nerve regeneration in goldfish. Brain Res. 216: 253-264.

Nishi, R., and D. K. Berg (1977) Dissociated ciliary ganglia neurons in vitro: Survival and synapse formation. Proc. Natl. Acad. Sci. U. S. A. 74: 5171-5175.

Pilar, G., L. Landmesser, and L. Burstein (1980) Competition for survival among developing ciliary ganglion cells. J. Neurophysiol. 43: 233-254.

Tuttle, J., J. Suszkiw, and M. Ard (1980) Long term survival and development of dissociated parasympathetic neurons in culture. Brain Res. 183: 161-180.

Varon, S., M. Manthorpe, and R. Adler (1979) Cholinergic neurotrophic factors. I. Survival, neurite outgrowth, and CAT activity in monolayer cultures from chick embryo ciliary ganglia. Brain Res. 173: 29-45.

Winick, M., and P. E. Greenberg (1965) Chemical control of sensory ganglia during a critical period of development. $\mathrm{Na}$ ture 205: 180-181. 